\title{
Calculating power integral bases by using relative power integral bases
}

\author{
István Gaál,; László Remete and Tímea Szabó \\ University of Debrecen, Mathematical Institute \\ H-4010 Debrecen Pf.12., Hungary \\ e-mail: igaal@science.unideb.hu,remetel42@gmail.com, \\ szabo.timea@science.unideb.hu
}

September 28, 2018

\begin{abstract}
Let $M \subset K$ be number fields. We consider the relation of relative power integral bases of $K$ over $M$ with absolute power integral bases of $K$ over $\mathbb{Q}$. We show how generators of absolute power integral bases can be calculated from generators of relative ones. We apply our ideas in infinite families of octic fields with quadratic subfields.
\end{abstract}

\section{Introduction: monogenity in the absolute and relative case}

Monogenity of number fields and the calculation of generators of power integral bases is a classical topic of algebraic number theory c.f. [17], [6]. We

*Research supported in part by K115479 from the Hungarian National Foundation for Scientific Research

2010 Mathematics Subject Classification: Primary 11R04; Secondary 11Y50

Key words and phrases: octic fields, relative quartic extension, power integral basis, relative power integral basis 
have general algorithms for calculating generators of power integral bases in lower degree number fields, [15], [13], 8], 11. We only have partial results for higher degree fields [3], [10], [9], [11], [4].

Let $K$ be an algebraic number field of degree $n$ with ring of integers $\mathbb{Z}_{K}$. This field is monogene if $\mathbb{Z}_{K}$ is a simple ring extension of $\mathbb{Z}$, that is there exist $\vartheta \in \mathbb{Z}_{K}$ such that $\mathbb{Z}_{K}=\mathbb{Z}[\vartheta]$. In this case $\left\{1, \vartheta, \ldots, \vartheta^{n-1}\right\}$ is an integral basis of $K$, called power integral basis. If $\alpha_{1}, \alpha_{2} \in \mathbb{Z}_{K}$ are related by $\alpha_{1} \pm \alpha_{2} \in \mathbb{Z}$ then the elements $\alpha_{1}, \alpha_{2}$ are called equivalent. These elements have the same indices (see below) and $\alpha_{1}$ generates a power integral basis of $K$ if and only if $\alpha_{2}$ does. Up to equivalence there are only finitely many generators of power integral bases of $K$.

We also considered monogenity and power integral bases in the relative case [5], 12], [16]. The element $\vartheta$ generates a relative power integral basis of

$K$ over the subfield $M$ if $\mathbb{Z}_{K}=\mathbb{Z}_{M}[\vartheta]\left(\mathbb{Z}_{M}\right.$ denotes the ring of integers of $M)$. In the relative case we call $\alpha_{1}, \alpha_{2} \in \mathbb{Z}_{K}$ equivalent if $\alpha_{1}+\varepsilon \alpha_{2} \in \mathbb{Z}_{M}$ for some unit $\varepsilon$ in $M$. These elements have the same relative indices (see below) and $\alpha_{1}$ generates a relative power integral basis of $K$ over $M$ if and only if $\alpha_{2}$ does. Up to equivalence there are only finitely many generators of relative power integral bases of $K$ over $M$.

In the present paper we describe the relation of the generators of relative power integral bases with the generators of absolute ones. We show how the generators of relative power integral bases can be used to calculate generators of absolute power integral bases.

The algorithm is especially simple if $M$ is a quadratic field. We apply our method to three infinite families of octic fields with imaginary quadratic subfields.

\section{From relative power integral bases to ab- solute ones}

Let $M$ be an algebraic number field of degree $m$ and $K$ an extension of $M$ with $[K: M]=k$. Then we have $[K: \mathbb{Q}]=k \cdot m$. Let $\mathcal{O}$ be either the ring of integers $\mathbb{Z}_{K}$ of $K$ or an order in $\mathbb{Z}_{K}$. Denote by $\mathbb{Z}_{M}$ the ring of integers of $M$. We assume that there exist a relative integral basis of $\mathcal{O}$ over $M$. (As we shall see in the following the existence of a power integral basis of $\mathcal{O}$ implies the existence of a relative power integral basis.) 
Denote by $D_{\mathcal{O}}$ and $D_{M}$ the discriminants of $\mathcal{O}$ and the subfield $M$, respectively. (In case $\mathcal{O}=\mathbb{Z}_{K}$ we have $D_{\mathcal{O}}=D_{K}$ where $D_{K}$ is the discriminant of the field $K$.) The index of a primitive element $\alpha$ of $\mathcal{O}$ with respect to the order $\mathcal{O}$ is

$$
I_{\mathcal{O}}(\alpha)=\frac{\sqrt{|D(\alpha)|}}{\sqrt{\left|D_{\mathcal{O}}\right|}}
$$

We also have

$$
I_{\mathcal{O}}(\alpha)=\left(\mathcal{O}^{+}: \mathbb{Z}[\alpha]^{+}\right)=\left(\mathcal{O}^{+}: \mathbb{Z}_{M}[\alpha]^{+}\right) \cdot\left(\mathbb{Z}_{M}[\alpha]^{+}: \mathbb{Z}[\alpha]^{+}\right),
$$

where the indices of the additive groups of the corresponding rings are calculated. The first factor is just the relative index of $\alpha$ :

$$
I_{\mathcal{O} / M}(\alpha)=\left(\mathcal{O}^{+}: \mathbb{Z}_{M}[\alpha]^{+}\right) .
$$

Denote by $D_{\mathcal{O} / M}$ the relative discriminant of $\mathcal{O}$ over $M$. As it is well known

$$
D_{\mathcal{O}}=N_{M / \mathbb{Q}}\left(D_{\mathcal{O} / M}\right) \cdot D_{M}^{[K: M]} .
$$

Denote by $\gamma^{(i)}$ the conjugates of any $\gamma \in M(i=1, \ldots, m)$. Let $\delta^{(i, j)}$ be the images of $\delta \in K$ under the automorphisms of $K$ leaving the conjugate field $M^{(i)}$ elementwise fixed $(j=1, \ldots, k)$. Then for any primitive element $\alpha \in \mathcal{O}$ we have

$$
\begin{gathered}
I_{\mathcal{O} / M}(\alpha)=\frac{\sqrt{\left|N_{M / \mathbb{Q}}\left(D_{\mathcal{O} / M}(\alpha)\right)\right|}}{\sqrt{\left|N_{M / \mathbb{Q}}\left(D_{\mathcal{O} / M}\right)\right|}}= \\
=\frac{1}{\sqrt{\left|N_{M / \mathbb{Q}}\left(D_{\mathcal{O} / M}\right)\right|}} \cdot \prod_{i=1}^{m} \prod_{1 \leq j_{1}<j_{2} \leq k}\left|\alpha^{\left(i, j_{1}\right)}-\alpha^{\left(i, j_{2}\right)}\right| .
\end{gathered}
$$

Further, by (11), (2), (3) and (44) we have

$$
\begin{gathered}
J(\alpha)=\left(\mathbb{Z}_{M}[\alpha]^{+}: \mathbb{Z}[\alpha]^{+}\right)= \\
=\frac{1}{\sqrt{\left|D_{M}\right|}[K: M]} \cdot \prod_{1 \leq i_{1}<i_{2} \leq m} \prod_{j_{1}=1}^{k} \prod_{j_{2}=1}^{k}\left|\alpha^{\left(i_{1}, j_{1}\right)}-\alpha^{\left(i_{2}, j_{2}\right)}\right| .
\end{gathered}
$$

The element $\alpha$ generates a power integral basis of $\mathcal{O}$ if and only if $I_{\mathcal{O}}(\alpha)=$ 1. Here we formulate the straightforward consequences of it, which will be very useful in our calculations in the following sections.

By (2),$I_{\mathcal{O}}(\alpha)=1$ can only be satisfied if both factors of (2) are equal to 1. Therefore, 
Proposition 1. A primitive element $\alpha \in \mathcal{O}$ generates a power integral basis of $\mathcal{O}$, if and only if

$$
I_{\mathcal{O} / M}(\alpha)=1
$$

and

$$
J(\alpha)=\left(\mathbb{Z}_{M}[\alpha]^{+}: \mathbb{Z}[\alpha]^{+}\right)=1
$$

Hence we have

Corollary 2. If $\alpha$ generates a power integral basis of $\mathcal{O}$, then it generates a relative power integral basis of $\mathcal{O}$ over $M$.

It is well known that generators or relative power integral bases are determined up equivalence, that is up to multiplication by a unit in $M$ and up to translation by element of $\mathbb{Z}_{M}$. Hence

Proposition 3. If a generates a power integral basis of $\mathcal{O}$, then

$$
\alpha=A+\varepsilon \cdot \alpha_{0},
$$

where $\alpha_{0}$ is a generator of a relative power integral basis of $\mathcal{O}$ over $M, \varepsilon$ is a unit in $M$ and $A \in \mathbb{Z}_{M}$.

Summarizing, in order to determine all generators of power integral bases of $\mathcal{O}$ we have to perform the following steps:

Step 1 Determine up to equivalence all generators $\alpha_{0} \in \mathcal{O}$ of relative power integral bases of $\mathcal{O}$ over $M$.

In other words, determine all elements $\alpha_{0} \in \mathcal{O}$ with relative index 1 :

$$
I_{\mathcal{O} / M}\left(\alpha_{0}\right)=1
$$

Note that if $\alpha_{0}$ has relative index 1 , then by means of equivalence any $\alpha$ of the form (7) also has relative index 1.

Step 2 Given $\alpha_{0}$ determine $\varepsilon$ and $A$ so that $\alpha$ of (7) has $J(\alpha)=1$.

Let $\mu_{1}=1, \mu_{2}, \ldots, \mu_{m}$ be an integral basis of $M$. Then the above $A$ can be represented in the form

$$
A=a_{1}+a_{2} \mu_{2}+\ldots+a_{k} \mu_{k}
$$


Since the (absolute) index is invariant under translation by an element of $\mathbb{Z}$, we have to calculate $a_{2}, \ldots, a_{m}$ of (8) up to sign. Step 2 means to determine $\varepsilon$ and $a_{2}, \ldots, a_{k}$ satisfying (6). In view of (5) this yields to solve an equation of degree $k^{2} m(m-1) / 2$ depending on $\varepsilon$ and $a_{2}, \ldots, a_{k}$.

This later task can became very complicated. However if $M$ is an imaginary quadratic field, then there are only finitely many units $\varepsilon$ in $M$ and we get a polynomial equation in one variable $a_{2}$. We shall apply our method in this case in the following examples of infinite parametric families of octic number fields.

\section{Simplest $D_{4}$ octics}

Recently B.K.Spearman and K.S.Williams [18] studied the family of simplest $D_{4}$ octics. Let $t>0$ be an integer parameter and $\vartheta$ a root of the polynomial $x^{8}+\left(t^{2}+2\right) x^{4}+1$. They showed that these polynomials are irreducible, and the field $K=\mathbb{Q}(\vartheta)$ has Galois group $D_{4}$. Assuming that $t^{2}+4$ is square free they calculated the discriminant of $K$ and gave an integral basis of $K$. By

$$
x^{8}+\left(t^{2}+2\right) x^{4}+1=\left(x^{4}+i t x^{2}+1\right)\left(x^{4}-i t x^{2}+1\right)
$$

$M=\mathbb{Q}(i)$ is a subfield of $K$.

Here we restrict ourselves to parameters of the form $t=2 T^{2}$. We explicitely describe all generators of relative power integral bases of the order $\mathcal{O}=\mathbb{Z}_{M}[\vartheta]$ over $\mathbb{Z}_{M}$. Moreover we show that the order $\mathcal{O}$ admits no power integral bases.

\subsection{Relative power integral bases in the family of $D_{4}$ octics}

Let $T$ be a nonzero integer parameter and $K$ the algebraic number field generated by a root $\vartheta$ of the polynomial $f(x)=x^{8}+\left(4 T^{4}+2\right) x^{4}+1$. Let $M=\mathbb{Q}(i)$. Denote by $\mathbb{Z}_{K}$ (resp. $\left.\mathbb{Z}_{M}\right)$ the ring of integers of $K$ (resp. $\left.M\right)$. Consider the order $\mathcal{O}=\mathbb{Z}_{M}[\vartheta]$ of $K$.

Our purpose is to explicitely determine all generators of relative power integral bases of $\mathcal{O}$ over $M$. Obviously, any $\alpha \in \mathcal{O}$ can be written in the form

$$
\alpha=A+X \vartheta+Y \vartheta^{2}+Z \vartheta^{3}
$$

with $A, X, Y, Z \in \mathbb{Z}_{M}$. 
Theorem 4. Assume $T>11$. Up to equivalence all generators of relative power integral bases of $\mathcal{O}$ over $\mathbb{Z}_{M}$ are given by

$$
\begin{gathered}
\alpha=\vartheta \\
\alpha=-2 i T^{2} \vartheta+\vartheta^{3}, \\
\alpha=\left(1+4 T^{4}\right) \vartheta \pm(1+i) T \vartheta^{2}+2 i T^{2} \vartheta^{3}, \\
\alpha= \pm(1+i) T \vartheta^{2}+\vartheta^{3} .
\end{gathered}
$$

Proof of Theorem 4. The octic polynomial $f(x)$ can be written as

$$
f(x)=\left(x^{4}+2 i T^{2} x^{2}+1\right)\left(x^{4}-2 i T^{2} x^{2}+1\right)
$$

hence the relative defining polynomial of $\vartheta$ over $M$ is $x^{4}-2 i T^{2} x^{2}+1$. In our proof we use the result of I.Gaál and M.Pohst [12] on power integral bases in relative quartic extensions (cf. also [6]).

According to [12] the coefficients $X, Y, Z \in \mathbb{Z}_{M}$ of $\alpha$ in (9) must satisfy

$$
\begin{aligned}
F(U, V)=\left(U-2 i T^{2} V\right)(U-2 V)(U+2 V) & =\varepsilon \\
Q_{1}(X, Y, Z)=X^{2}-2 i T^{2} Y^{2}+4 i T^{2} X Z+\left(1-4 T^{4}\right) Z^{2} & =U, \\
Q_{2}(X, Y, Z)=Y^{2}-X Z-2 i T^{2} Z^{2} & =V,
\end{aligned}
$$

with a unit $\varepsilon$ of $M$ and with $U, V \in \mathbb{Z}_{M}$. We have to determine the solutions $U, V \in \mathbb{Z}_{M}$ of the first equation and for all pairs $U, V$ to calculate the corresponding solutions $X, Y, Z$ of the second and third equations. By the first equation we have $U-2 V=\varepsilon_{1}$ and $U+2 V=\varepsilon_{2}$ with units $\varepsilon_{1}, \varepsilon_{2} \in M$. Therefore $4 V=\varepsilon_{2}-\varepsilon_{1}$. Since all units in $M$ are $\pm 1, \pm i$, the only $V \in \mathbb{Z}_{M}$ satisfying this equation is $V=0$. Hence $U$ is again a unit in $M$. Following the method of [12] we set

$$
Q_{0}(X, Y, Z)=U Q_{2}(X, Y, Z)-V Q_{1}(X, Y, Z)=0 .
$$

Using standard arguments described in [12] we can parametrize $X, Y, Z$ with parameters $P, Q \in \mathbb{Z}_{M}$ so that up to a unit factor we get

$$
X=P^{2}-2 i T^{2} Q^{2}, \quad Y=P Q, \quad Z=Q^{2} .
$$


Substituting the formulas (10) into $Q_{1}(X, Y, Z)=U$ we obtain a quartic relative Thue equation over $M$ :

$$
P^{4}-2 i T^{2} P^{2} Q^{2}+Q^{4}=\varepsilon,
$$

with a unit $\varepsilon$ in $M$. This equation can be written in the form

$$
P^{4}-((1+i) T)^{2} P^{2} Q^{2}+Q^{4}=\varepsilon,
$$

therefore we may apply the results of V.Zielger [19] on the solution of this equation by taking $t=(1+i) T$ as parameter. Theorem 2 of [19] implies that, assuming $\left|t^{2}\right|>245$, that is $|T|>11$, up to unit factors of $M$ all solutions of (11) are

$$
(P, Q)=(1,0),(0,1),(1, \pm(1+i) T),((1+i) T, \pm 1) .
$$

Substituting these vales of $(P, Q)$ into (10) we obtain the possible triplets:

\begin{tabular}{|c|c|c|c|}
\hline & $x$ & $y$ & $z$ \\
\hline Case 1 & 1 & 0 & 0 \\
\hline Case 2 & $-2 i T^{2}$ & 0 & 1 \\
\hline Case 3 & $1+4 T^{4}$ & $\pm(1+i) T$ & $2 i T^{2}$ \\
\hline Case 4 & 0 & $\pm(1+i) T$ & 1 \\
\hline
\end{tabular}

This proves Theorem 4 .

\subsection{Power integral bases in the family of $D_{4}$ octics}

Despite of the promising result on relative power integral bases we have

Theorem 5. For $|T|>11$ the order $\mathcal{O}$ admits no power integral bases.

Proof of Theorem 5. In view of (17) a generator $\alpha$ of a power integral basis of $\mathcal{O}$ must be of the form $\alpha=a_{1}+a_{2} i+\varepsilon \alpha_{0}$ where $a_{1}, a_{2} \in \mathbb{Z}, \varepsilon= \pm 1, \pm i$ and the possibe values of $\alpha_{0}$ are listed in Theorem 4. Any $\alpha$ of the above form has relative index $I_{\mathcal{O} / M}(\alpha)=1$. The index of $\alpha$ is independent of $a_{1}$ and it is sufficient to determine $\alpha$ up to sign. Therefore we have to consider the possible values of $\alpha_{0}$ and for $\varepsilon=1, \varepsilon=i$ and we have to calculate $J(\alpha)$. We have $D_{M}=-4$ hence

$$
J(\alpha)=\frac{1}{2^{4}} \prod_{j_{1}=1}^{4} \prod_{j_{2}=1}^{4}\left|\alpha^{\left(1, j_{1}\right)}-\alpha^{\left(2, j_{2}\right)}\right| .
$$


In Case 1 we get

$J(\alpha)=2^{4} \cdot\left|\left(4 T^{2} a_{2}^{2}-1+4 a_{2}^{2}\right)\left(4 T^{2} a_{2}^{2}+1-4 a_{2}^{2}\right)\left(T^{8}+8 a_{2}^{4} T^{4}+16 a_{2}^{8}+16 a_{2}^{4}\right)\right|$.

Hence $J(\alpha)$ is divisible by $2^{4}$, yielding that $\alpha$ can not be a generator of a power integral basis.

In the other cases we got much more complicated formulas, but in each case $J(\alpha)$ is divisible by $2^{4}$.

\subsection{Remarks on the numerical calculations}

All calculations involved in the proof of Theorem 5 were performed in Maple [2] under Linux. $J(\alpha)$ is a polynomial with integer coefficients of degree 16 in $a_{2}$, depending also heavily on the parameter $T$. We used symmetric polynomials several times to simplify the formulas. Without being very careful the formulas became extremely complicated and Maple broke down in lack of memory space. Using careful approach all calculations took less than 2 minutes.

\section{Composites of imaginary quadratic fields and pure quartic fields}

In a recent paper [14] we considered number fields of type $K=\mathbb{Q}(\sqrt[4]{m}, i \sqrt{d})$ for $d=3,7,11,19,43,67,163$ and for $1<m \leq 5000, m \equiv 2,3(\bmod 4)$ with $(d, m)=1$. Set $\xi=\sqrt[4]{m}, \omega=(1+i \sqrt{d}) / 2$, then

$$
\left\{1, \xi, \xi^{2}, \xi^{3}, \omega, \omega \xi, \omega \xi^{2}, \omega \xi^{3}\right\}
$$

is an integral basis of $K$ and $\left\{1, \xi, \xi^{2}, \xi^{3}\right\}$ is a relative integral basis of $K$ over $M=\mathbb{Q}(i \sqrt{d})$. In [14] we described all generators

$$
\alpha=A+X \xi+Y \xi^{2}+Z \xi^{3}
$$

of relative power integral bases of $K$ over $M$ with $A, X, Y, Z \in \mathbb{Z}_{M}$ and $\max (\overline{|X|}, \overline{|Y|}, \overline{|Z|})<10^{500}$ (here $\overline{|X|}$ denotes the size of $X$ that is the maximum absolute value of its conjugates). The problem lead us to a quartic relative binomial Thue equation. Using the algorithm of [7] we calculated the "small" solutions of this equation which resulted Theorem 3 of [14]. Note 
that according to our experience these equations never have "large" solutions hence our list contains all solutions with high probability. Further, calculating the "small" solutions was the only way to deal with thousands of relative Thue equations.

Using the ideas of Section 2 we tested if there exist generators of power integral bases of $K$ over $\mathbb{Q}$ corresponding to the relative power integral bases found in Theorem 3 of [14]. We have

Theorem 6. Let $d=3,7,11,19,43,67,163$ and $1<m \leq 5000$ with $m \equiv$ $2,3(\bmod 4)$ and $(d, m)=1$. Then the number field $K=\mathbb{Q}(\sqrt[4]{m}, i \sqrt{d})$ does not admit any generators of power integral bases of the form

$$
\alpha=A+\varepsilon\left(X \xi+Y \xi^{2}+Z \xi^{3}\right)
$$

where $A \in \mathbb{Z}_{M}$, $\varepsilon$ a unit in $M$ and $X, Y, Z \in \mathbb{Z}_{M}$ with

$$
\max (\overline{|X|}, \overline{|Y|}, \overline{|Z|})<10^{500} \text {. }
$$

Proof of Theorem 6. For all possible values of $X, Y, Z$ listed in Theorem 3 of 14 and for all possible unit $\varepsilon$ in $M$ we set $A=a_{1}+a_{2} \omega$. We calculated $J(\alpha)$ which is a polynomial in $a_{2}$ with integral coefficients of degree 16 . In each case we found that $J(\alpha)= \pm 1$ is not solvable for $a_{2}$ in integers. Calculation with polynomials with integer coefficients was very fast, the whole calculation took a few seconds.

\section{Parametric families of quartic extensions of imaginary quadratic fields}

In [16] we calculated generators of relative power integral bases in infinite parametric families of orders of certain octic fields. Here in two of these families we check if there exist corresponding generators of (absolute) power integral bases. The challenge of these examples is that $J(\alpha)$ depends not only on $a_{2}$ but also on the quadratic field and the parameter of the family.

I. Let $d>0$ be an integer, $-d \equiv 2,3(\bmod 4)$ and set $M=\mathbb{Q}(i \sqrt{d})$. Let $t \in \mathbb{Z}_{M}$ be a parameter and let $\xi$ be a root of the polynomial

$$
f(x)=x^{4}-t^{2} x^{2}+1 .
$$


Consider $\mathcal{O}=\mathbb{Z}_{M}[\xi]$. In [16] we showed that for $|t|>245$ up to equivalence there are five generators of relative power integral bases of $\mathcal{O}$ over $\mathbb{Z}_{M}$, namely $\alpha_{0}=\xi,-t^{2} \xi+\xi^{3},\left(1-t^{4}\right) \xi+t \xi^{2}+t^{2} \xi^{3},\left(1-t^{4}\right) \xi-t \xi^{2}+t^{2} \xi^{3}, t \xi^{2}+\xi^{3},-t \xi^{2}+\xi^{3}$.

We have

Theorem 7. Under the above conditions for $|t|>245$ the order $\mathcal{O}$ admits no power integral bases.

Proof of Theorem [7. Denote by $\alpha_{0}$ a possible generator of a relative power integral basis of $\mathcal{O}$ over $\mathbb{Z}_{M}$, say

$$
\alpha_{0}=\left(1-t^{4}\right) \xi+t \xi^{2}+t^{2} \xi^{3}
$$

where $t=t_{1}+t_{2} i \sqrt{d}$ is the parameter $\left(t_{1}, t_{2} \in \mathbb{Z}\right)$. Note that since the minimal polynomial of $\xi$ over $\mathbb{Z}_{M}$ depends on the parameter $t \in \mathbb{Z}_{M}$, hence $\xi$ depends on $t$ and also on $d$. We let $\varepsilon= \pm 1$ and represent $\alpha$ in the form

$$
\alpha=a_{1}+a_{2} i \sqrt{d}+\varepsilon \alpha_{0}
$$

with $a_{1}, a_{2} \in \mathbb{Z}$. Then we calculate $J(\alpha)$. This is a very complicated polynomial of degree 16 depending not only on $a_{2}$ but also on $t_{1}, t_{2}, d$. Using symmetric polynomials and simplifying the formulas very carefully, we obtain that $J(\alpha)$ is disvisible by 16 . Therefore there are no generators of power integral bases of $\mathcal{O}$ corresponding to $\alpha_{0}$. The proof runs the same way for the other four candidates of $\alpha_{0}$, as well. The Maple calculation took 10-60 seconds per case.

II. Let again $d>0$ be an integer, $-d \equiv 2,3(\bmod 4), M=\mathbb{Q}(i \sqrt{d})$. Let $t \in \mathbb{Z}_{M}$ be a parameter and let $\xi$ be a root of the polynomial

$$
f(x)=x^{4}-4 t x^{3}+(6 t+2) x^{2}+4 t x+1 .
$$

Let $\mathcal{O}=\mathbb{Z}_{M}[\xi]$. According to [16] for $|t|>1544803$ up to equivalence there are two generators of power integral bases of $\mathcal{O}$ over $\mathbb{Z}_{M}$, namely

$$
\alpha_{0}=\xi,(6 t+2) \xi-4 t \xi^{2}+\xi^{3} \text {. }
$$

We have

Theorem 8. Under the above conditions for $|t|>1544803$ the order $\mathcal{O}$ admits no power integral bases.

The proof of this statement is similar to the proof of Theorem 7 , 


\section{References}

[1] Y.Bilu, I.Gaál and K.Györy, Index form equations in sextic fields: a hard computation, Acta Arithm., 115.1 (2004), 85-96.

[2] B.W.Char, K.O.Geddes, G.H.Gonnet, M.B.Monagan, S.M.Watt (eds.) MAPLE, Reference Manual, Watcom Publications, Waterloo, Canada, 1988.

[3] I.Gaál, Power integral bases in composits of number fields, Canad. Math. Bull., 41(1998), 158-161.

[4] I.Gaál, Solving index form equations in fields of degree nine with cubic subfields, J.Symbolic Comput., 30(2000), 181-193.

[5] I.Gaál, Power integral bases in cubic relative extensions, Experimental Math., 10(2001), 133-139.

[6] I.Gaál, Diophantine equations and power integral bases, Boston, Birkhäuser, 2002.

[7] I.Gaál, Calculating "small" solutions of relative Thue equations, Experimantal Math. 24(2015), 1-8.

[8] I.Gaál and K.Györy, Index form equations in quintic fields, Acta Arith., 89(1999), 379-396.

[9] I.Gaál, Computing all power integral bases in orders of totally real cyclic sextic number fields, Math. Comp., 65(1996), 801-822.

[10] I.Gaál, P.Olajos and M.Pohst, Power integral bases in orders of composits of number fields, Experimental Math., 11(2002), 87-90.

[11] I.Gaál and M.Pohst, On the resolution of index form equations in sextic

fields with an imaginary quadratic subfield, J.Symbolic Comp, 22(1996), 425-434. 
[12] I.Gaál and M.Pohst, On the resolution of index form equations in relative quartic extensions, J.Number Theory, 85(2000), 201-219.

[13] I.Gaál, A.Pethő and M.Pohst, Simultaneous representation of integers by a pair of ternary quadratic forms - with an application to index form equations in quartic number fields, J.Number Theory, 57(1996), 90-104.

[14] I.Gaál, L.Remete and T.Szabó, Calculating power integral bases by solving relative Thue equations, Tatra Mt. Math. Publ. 59 (2014), 7992.

[15] I.Gaál and N.Schulte, Computing all power integral bases of cubic number fields, Math. Comput., 53(1989), 689-696.

[16] I.Gaál and T.Szabó, Relative power integral bases in infinite families of quartic extensions of quadratic field, JP Journal of Algebra, Number Theory and Applications, 29(2013), 31-43.

[17] W.Narkiewicz, Elementary and Analytic Theory of Algebraic Numbers, Second Edition, Springer, 1974.

[18] B.K.Spearman and K.S.Williams, The simplest $D_{4}$ octics, Int. J. Algebra, 2(2008), 79-89.

[19] V.Ziegler, On a family of relative quartic Thue inequalities, J.Number Theory, 120(2006), 303-325. 